anvertraut $\mathrm{zu}$ werden, der dafür am besten geeignet ist « $;{ }^{11 \mathrm{I}} \mathrm{zum}$ anderen dürfte Ergebnis allein des Bestehens dieser zusätzlichen Sorgerechtsalternative eine verstärkte Begründung des vorzugswürdigeren gemeinsamen Sorgerechts sein, ${ }^{112} \mathrm{da}$ damit die bisherige Prämierung unkooperativen Verhaltens von Müttern entfiele. Schließlich würde damit jenen Männern gesellschaftliche Anerkennung zuteil, die gerade in Überwindung geschlechtsspezifischer Rollenzuweisungen durch eine aktive Vaterschaft den seit Jahrzehnten eingeforderten »neuen Mann « auch leben. ${ }^{113}$ Frauen sollten aus den bitteren Erfahrungen mit Rollenzuweisungen für Sorgerechtskonzeptionen offen sein, die nicht schematisch, sondern flexibel sind. Auch auf dieser gesellschaftlich übergreifenden Ebene ist im Interesse des Kindeswohls nicht ideologisierende Geschlechterkampfkonfrontation, sondern brückenschlagende Kooperation in dem Bewusstsein gefragt, dass mit der Vielgestaltigkeit des Menschen eine breite Konfliktlösungspalette zu korrespondieren hat.

\title{
Bernd Belina \\ Fighting Crime als Lebensaufgabe, Culture War und Kontrolle der Überflüssigen
}

\section{Anmerkungen zur Kriminalpolitik in den USA *}

Die eigentliche Arbeit der Chefs lokaler Polizeien in den USA ist trotz weiterreichender Kompetenzen mit der ihrer Kollegen hierzulande durchaus vergleichbar. Was ihren Status angeht, scheinen die Chiefs in mancherlei Hinsicht aber mehr mit hiesigen Trainern im professionellen Fußball gemeinsam zu haben: In beiden Fällen geht es um gut bezahlte Posten im Scheinwerferlicht der (vor allem lokalen) Öffentlichkeit. In beiden Fällen existiert nur eine recht begrenzte Anzahl wirklich verlockender Posten $(36$ Vereine in den Bundesligen I und 2 bzw. etwa ebenso viele Police Departments mit einer Personalstärke von über r.000 Polizisten, vgl. Hickman, Reaves 200I). In beiden Fällen beschränkt sich - in Ermangelung einer formalen Ausbildung - die Qualifikation der Bewerber häufig darauf, das Berufsfeld >von der Pike auf < (als Spieler bzw. Polizist) erlernt zu haben (Bouza I990: 78). Und in beiden Fällen gibt es Stars mit Vorbildfunktion, die mitunter sogar zu Buchautoren werden, wie der ehemalige Chef der New Yorker Polizei, William Bratton (1998), von dem noch die Rede sein wird, oder Anthony V. Bouza (1990, 200I), ehemals Commissioner in Minneapolis. (Zu den literarischen Werken von Fußballtrainern vgl. die Sammelbesprechung bei Fischer, Roth 1998: I 54-162.)

In diesem Beitrag soll es um das 1999 erschienene Buch eines der schillerndsten Helden der U.S.-amerikanischen Polizeiarbeit der letzten Jahre gehen, um The Crime

I I Henrich, FamRZ 2003, S. 359.

I 2 Darauf deutet auch das Ergebnis des von R. Proksch, Begleitforschung zur Umsetzung der Neuregelungen zur Reform des Kindschaftsrechts, 2. Zwischenbericht (Juli 200I), erstellten Zwischenberichts (auf S. 2 I 8 ff.) hin. Sie auch ders. in Kind-Prax 2003, S. 3 ff.

I 3 Der Vater im Ausgangsverfahren vor dem AG Groß-Gerau etwa hatte das Kind etwa acht Monate allein versorgt, später nach dem Herausgabeverlangen der Mutter auch während deren Krankenhausaufenthalts.

* Anhand und zugleich Besprechung von: Jack Maple (with Chris Mitchell): The Crime Fighter - Putting the Bad Guys out of Business. New York 1999: Doubleday. 260 Seiten. 
Fighter von Jack Maple. Der schon dank seines extravaganten Kleidungsstils (Al

Capone-Hut, Fliege, zweifarbige Halbschuhe) aus dem Rahmen fallende Maple hatte, ausgestattet nur mit dem Äquivalent eines High School Abschlusses, eine typische Karriere durch die Dienstgrade der New Yorker Transit Police hinter sich, bevor er es unter Chief William Bratton zunächst bei der Transit Police, I994 dann - nach einer kurzen gemeinsamen Zeit in Boston - beim New York Police Department (NYPD) bis zum stellvertretenden Polizeichef brachte. Als Bratton nach zwei Jahren Dauerstreit mit Bürgermeister Rudolph Giuliani 1996 zurücktrat, verließ wenige Tage später auch Maple das NYPD und gründete zusammen mit John Linder, der ebenfalls zum engen Zirkel um Bratton gehört hatte, eine Beratungsfirma.

Auch wenn es Maple, der im August 200 I an Dickdarmkrebs verstarb, selbst nie bis zum Polizeichef brachte, tragen heute doch zahlreiche Polizeien deutlich seine Handschrift, was zwei Gründe hat. Zum einen galt er als eigentlicher - da für Taktik und Strategie zuständiger-Vater der hochgelobten New Yorker Erfolgsgeschichte seit 1994, die unter dem Label zero tolerance bekannt wurde. Da »die größten police departments des Landes eine Bedeutung [besitzen], die weit über ihre reine Größe hinausgeht « und »definieren, was policing in den USA bedeutet «" (Harring, Ray I 999: 64), wird die Entwicklung des NYPD im Rest der USA bzw. Welt mit Interesse verfolgt und z. T. kopiert (zur weltweiten Verbreitung vgl. Wacquant 2000; Smith 2001; Belina, Helms 2003).Zum anderen war Maple nach seinem Wirken in New York als »superstar law enforcement consultant « (Zurawik 2002) u. a. in New Orleans, Newark, Philadelphia, Birmingham (Alabama) und Baltimore tätig. ${ }^{2}$

Bei Jack Maple handelt es sich also um einen einflussreichen Protagonisten der USamerikanischen Kriminalpolitik der i990er Jahre. Aus diesem Grund scheint es angebracht, sich nicht nur mit dem eigentlichen Inhalt des Buches, d.h. mit den von Maple verfolgten und angepriesenen Strategien zu beschäftigen, was im ersten Teil dieses Textes geschehen wird. Darüber hinaus soll auch gefragt werden, wie der Autor eigentlich die Welt sieht, in der er als Crime Fighter tätig ist (Teil II.) und warum diese Sicht auf die Welt auf so viel Zuspruch traf und trifft (Teil III.).

\section{Die Strategien des Jack Maple}

In den elf Kapiteln des Buches legt Maple seine Ansichten zur Polizeiarbeit dar. Grundlegend sind dabei vier Grundprinzipien (32):3 I. akkurate, zeitnahe Informationen; 2. schneller Einsatz; 3. effektive Taktik; 4. unnachgiebige Erfolgskontrolle und Beurteilung.

$\mathrm{Zu}$ I.: In technologischer Hinsicht war die Einführung einer zeitnahen Erfassung der Kriminalstatistik und ihre exakte Differenzierung nach Delikten und Tatorten das

I Übersetzung englischer Zitate: B. B.

2 So engagierte in Baltimore der spätere Bürgermeister, der im Wahlkampf ganz auf zero tolerance gesetzt hatte, Maple und Linder bereits am Tag nach seinem Sieg bei der Vorwahl der Demokratischen Partei (womit er im demokratisch dominierten Baltimore als nächster Bürgermeister praktisch fest stand). Der für \$ 2.000/ Tag erstellte »Plan zur dramatischen Reduzierung der Kriminalität in Baltimore (veröffentlicht unter den Namen von Bürgermeister und Polizeichef als O’Malley, Norris 2000) wurde in der Folge zur Richtschnur für das Baltimore Police Department (BPD), das außerdem mit Edward Norris einen ehemaligen Vertrauten Maples aus New York als neuen stellvertretenden Polizeichef bekam. Neuer Commissioner wurde ein altgedienter Beamter des BPD, der nach eigenen Angaben Maples Buch drei mal gelesen hatte. Nachdem er aber bereits nach weniger als zwei Monaten zurücktrat, weil er sich durch die Vorgaben des Maple-LinderPlans in seinen Arbeitsmöglichkeiten zu stark eingeschränkt sah und von Norris beerbt wurde, herrschte im BPD nach Einschätzung von Beobachtern endgültig ein "New York State of Mind « (Jones 2002; Wilber 200I; Rath 2000).

3 Seitenangaben beziehen sich immer auf Maple (1999) 
zentrale Element der Reformen des NYPD. Die Daten lagen dank des Computersystems »Compstat « (»computer statistics«) oder »Comstat (»comparative statistics «) - welcher Name zuerst da war, weiß niemand mehr genau (33) - zunächst wöchentlich, später täglich vor und bildeten die Grundlage von Strategieentwicklung, Einsatzplanung und der mittlerweile legendären grillings (vgl. Bratton I 998: 233-239). Auch betont Maple die Bedeutung der Umsetzung dieser Daten in thematische Karten: »Karten sind Zahlen oder Geschichten weit überlegen« (I05). Damit spricht er eine in der jüngeren Entwicklung der Humangeographie in den Mittelpunkt des Interesses gerückte Einsicht an, nämlich das »ein räumlicher Kode [...] meist von fast irreduzibler Simplizität« (Hard 1987: I33) ist. Mit Hilfe räumlicher Abstraktionen lassen sich komplexe Sachverhalte wesentlich einfacher kommunizieren (Klüter 1986). An die Stelle der (komplexen) Frage: Was ist ein und wie kommt es zu Verbrechen? tritt die (wesentlich einfachere): Wo hat es stattgefunden und warum da? In diesem Sinne verweisen Karten laut Maple auf die »dahinterliegenden Gründe« (105). Damit sind keine Erklärungen gemeint, sondern Fragen wie: »Gibt es da einen Crack Markt? Eine Bushaltestelle? Ein Einkaufszentrum?« (I05). Aus den »dahinterliegenden Gründen «, ehemals eine typische Formulierung von (liberalen und linken) Akteuren, die Kriminalität mit strukturellen Ursachen wie Armut und Rassismus erklären wollten (Beckett I997: 72), wird so die gebaute Umwelt (zur Kritik der Verräumlichung von Kriminalität und der damit einhergehenden Abstraktion vgl. Belina 2000; 2000a).

$\mathrm{Zu}$ 2. Die relativ simple Idee ist hierbei, die Einsatzplanung auf Basis der Kartierung zu betreiben und Polizisten dann und dort einsetzen, wann und wo die meisten Verbrechen erfasst wurden.

$\mathrm{Zu}$ 3. Unter diesem Punkt zählt Maple insgesamt sieben Taktiken auf, die alle den drei übergeordneten Zielen dienen sollen, Verbrecher I. so früh wie möglich auf ihrer Tour festzunehmen, 2. sie dazu zu bringen, ihre schlimmsten Verhaltensweisen abzulegen, und 3. ihren ihre Waffen abzunehmen (I I I).

Die wohl wichtigste und am bekanntesten gewordene Taktik ist deren erste: »Quality of life enforcement « (I II-I6I; im weiteren QOL). Maple vertritt die These, dass die Verfolgung auch kleinster Delikte, wie Urinieren oder Alkoholkonsum in der Öffentlichkeit, Abspielen zu lauter Radios oder Schwarzfahren »schwerere Verbrechen verhindern [kann], wenn jede Kontrolle oder jede Verhaftung als eine Gelegenheit behandelt wird, einen Flüchtigen einzusperren oder Informationen über ungelöste Verbrechen zu erlangen« (I I I). Für die Debatten über die Polizeiarbeit in New York Mitte der I 990er Jahre ist dies m. E. als eine Klarstellung zu betrachten, die einer beliebten und auch massenmedial verbreiteten Lesart zuwiderläuft: Die kleinen Vergehen sollen demnach verfolgt werden, um einen (d.h.: irgendeinen) Anlass zu haben, Leute, die irgendwie verdächtig aussehen oder wirken, kontrollieren und verhören zu können. Das ist etwas völlig anders, als der üblicherweise behauptete Grund für die Verfolgung von QOL-Vergehen, dass nämlich ein kausaler Zusammenhang zwischen dem durch die kleinen Vergehen ruinierten Erscheinungsbild einer Gegend und den dort begangenen schweren Verbrechen bestehe. Dieser Zusammenhang firmiert bekanntlich unter dem Schlagwort Broken Windows und wird von seinen Erfindern auch in aller Deutlichkeit formuliert: »ernsthafte Straßenkriminalität gedeiht in Gegenden, in denen unordentliches Verhalten unkontrolliert durchgeht « (Wilson, Kelling I982: 34). Besonders anschaulich stellen diesen behaupteten Zusammenhang George Kelling, sein wichtigster Vertreter in wissenschaftlichen Kreisen, und William Bratton dar: »Unordnung $\rightarrow$ Furcht der Bürger $\rightarrow$ Rückzug (physisch \& sozial) $\rightarrow$ Zunahme schlechter Verhaltensweisen $\rightarrow$ Zunahme des Verbrechens $\rightarrow$ Kreislauf des Niedergangs « (I998: I 2 I9). Gegen eine solche »mystische Verbindung 
brechen« (I 54) polemisiert Maple ganz explizit:

Vergewaltiger und Mörder ziehen nicht in eine andere Stadt, wenn sie sehen, dass die Graffitis aus der U-Bahn verschwinden. Der normale Squeegee Man [Bettler, der unaufgefordert die Fenster an Ampeln haltender PKW putzt; erstes Ziel der QOL Polizeiarbeit in New York; B. B.] fängt nicht an, bezahlte Mordaufträge anzunehmen, wenn er eine gestiegene Toleranz fürs unerwünschte Fensterputzen auf der Straße wahrnimmt. Betteln verwandelt keine Nachbarschaft in Murder Central. (I 54 f.)

Stattdessen betont er: »QOL Polizeiarbeit reduziert Verbrechen, weil es der Polizei erlaubt, die Gauner zu schnappen, wenn sie nicht im Dienst sind « (I55). Deshalb kann es laut Maple auch nicht darum gehen, alle Verstöße gegen die Lebensqualität immer und überall zu verfolgen, sondern selektiv nur in den am stärksten kriminalitätsbelasteten Gegenden und nur gegen potentielle Flüchtige oder Informanten. Dies ist laut Maple auch der Grund, warum die von ihm vertretene und in New York zu seiner Zeit angewandet Strategie nicht zero tolerance sei, da dies null Toleranz gegen jedes Fehlverhalten überall bedeuten würde. Maple beklagt sich später im Buch vielmehr darüber, dass ihm überall der Ruf vorauseilt, für zero tolerance und damit auch für Polizeiübergriffe zu stehen (2 I 2 f.). Stattdessen stellt er klar: »Zero tolerance ist schlechte Polizeiarbeit und eine schlechte Taktik« (2I3).

Was QOL-policing im New York Brattons und Maples in der Praxis bedeutete, zeigen Jeffrey Fagan and Garth Davies (2000) anhand der Auswertung der Polizeikontrollen aus jener Zeit. Dabei kommen sie zu dem Ergebnis, dass »eine massive Zunahme von Festnahmen auf Grund von Vergehen [= misdemeanors][zu verzeichnen war], zugleich aber ihre Qualität deutlich abnahm und sie vor Gericht seltener zu halten waren « (ebd.: 476). Die Autoren folgern, dass es bei der Strategie offenbar »um die Kontrolle von armen Leuten in armen Gegenden « $(e b d:$ : 457) ging. Diese Empirie verweist auf eine Funktion der zero tolerance Rhetorik: Mit ihr wird davon abgesehen, dass es nicht um Kontrolle von jedem und überall, sondern um diejenige von äußerlich Auffälligen an bestimmten Orten geht, d.h. in den USA von Afroamerikanern und Hispanics in den Ghettos und Barrios.

In diesem Sinne ist die erste Hälfte von Maples Taktik Nummer Eins, d.h. die überproportionale Kontrolle von Minderheiten, auch keine ganz neue Idee, sondern vor allem die konsequente Anwendung einer in der US-amerikanischen Polizeiarbeit wohl bekannten Praxis. Seit dem Supreme Court Urteil Terry v. Ohio (1968) sind Kontrollen und Identitätsfeststellungen ohne angemessenen Anfangsverdacht untersagt (Gebring I999: IOI-IO5). Deshalb sind Polizisten gezwungen, irgendeinen Grund zu finden, wenn sie Personen anhalten wollten, die sich außer einem abweichenden Äußeren oder der falschen Hautfarbe nichts zu Schulden haben kommen lassen. So entstanden zunächst für Autofahrer die pretext stops (Cole I 999: 34-4I), also Kontrollen unter einem Vorwand. Neu an Maples Taktik ist vor allem die zweite

4 Maples Ziel, das Auffinden von illegalen Waffen und Flüchtigen, bezeichnet Bratton als »unvorhergesehenes Nebenprodukt « (1998: I 54), Kelling als »eine neue Dimension der >Broken Windowsく Idee« (200 I: I 28), die zu dem eigentlichen Zweck hinzukomme. Beide halten dabei jedoch an ihrer ursprünglichen Formulierung als primärem Grund für QOL Polizeiarbeit fest. Für den prominentesten Fürsprecher der New Yorker Polizeistrategie in Kreisen der deutschsprachigen Kriminologie, Henner Hess, ist die Praxis der New Yorker Transit Police, die unter Bratton in der Zeit von 1990-1 992 bereits ähnlich vorging, ein »guter Beleg für die Broken Windows-Theorie« (I999: 45), weil bei der Jagd auf Schwarzfahrer auch Flüchtige und Waffen gefunden wurden (ebd.). Zu dieser Verwechslung von Broken Windows (wonach die Verfolgung von Schwarzfahrern über das dadurch gesteigerte Erscheinungsbild der U-Bahn dazu führen müsste, dass weniger Diebstähle und Morde passieren, weil Diebe und Räuber sich unwohl fühlen - eine absurde Vorstellung) und ihrem »Nebeneffekt « gelangt er, obwohl er beides in demselben Text analytisch trennt und QOL-policing als »einerseits Selbstzweck [also: Broken Windows; B. B.], andererseits das Schleppnetz, in dem sich schwerere Kriminelle fangen« (ebd.: 46) bezeichnet. 
Hälfte, d. h. dass sich in New York an diese rassistisch motivierten Kontrollen auch noch genaue Durchsuchungen und intensive Verhöre anschlossen. Zum Thema QOLpolicing kann also mit Sidney Harring und Gerda Ray geschlossen werden, dass dadurch Terry Stops - also Kontrollen ohne Anfangsverdacht - noch weiter verbreitet werden und so »die schlechte Polizeiarbeit der I960er und I970er die gute Polizeiarbeit der I990er « wird (1999: 79).

$\mathrm{Zu}$ 4.: Hier betont Maple vor allem die Bedeutung der Kontrolle der Arbeit aller Beteiligten auf allen Ebenen der Polizeihierarchie sowie der Einrichtung einer »cold case squad « ( ( 82), die sich alle ungelöst gebliebenen Fälle von Bedeutung noch einmal vornimmt.

Weiterhin wendet sich Maple dem Thema Drogen zu. Dabei geht es vor allem um die I995 von Bratton und Maple geplante Operation Juggernaut, die nie verwirklicht wurde. Mit ihr wollten sie »das Gelände von den Dealern zurückgewinnen und niemals wieder ein Zoll zurückweichen« (200) und damit »innerhalb eines Jahres die Kriminalität auslöschen [sic!] « (200). Der Weg dorthin sollte über die Stationierung zusätzlicher Polizeieinheiten führen, um »eine Drogengang nach der anderen hochzunehmen, vom Straßendealer über die mittlere Ebene bis zum Chef des Ganzen und darüber hinaus« (200).

Innerhalb der Debatte um den war on drugs positioniert sich Maple damit eindeutig: Auch er geht davon aus, dass die seit den i $980 e r$ Jahren verfolgte sangebotsorientierter Strategie, d.h. die strafrechtliche Verfolgung von Dealern, Drogenringen und Drogennutzern, und nicht eine snachfrageorientierte Strategie, die auf Drogenhilfe und Therapie setzt, der Weg zur >Lösung ‘ des >Drogenproblems` sei (zum war on drugs und den Debatten um seine Erfolglosigkeit vgl. Beckett I997: 44-6I; Lock, Timberlake, Rasinski 2002; Redmond 200I). Dass sich damit an der Sucht (und damit Nachfrage nach illegalen Drogen) nichts ändert, dass also das >Drogenproblem natürlich weiter bestehen würde, findet in dieser siegesgewissen Rhetorik keine Berücksichtigung. Zu Pass kommt Maple dabei, dass die Operation niemals Wirklichkeit wurde. 5 Wie Vieles, das in seiner New Yorker Zeit ohne Erfolg blieb, macht er auch hier City Hall, mithin den als medien- und machtbesessen charakterisierten Bürgermeister Giuliani, verantwortlich (200).

Abschließend räumt Maple ein, dass sie in New York nicht genug Zeit hatten, etwas gegen die »übliche Einstellung unter Cops, in der die allgemeine Öffentlichkeit bestenfalls ein Ärgernis und schlimmstenfalls ein Feind [ist] « (2 I I) zu tun. Um derartige, von ihm für notwendig erachtete Reformen voranzutreiben, plädiert er dafür, dass jede Polizei 5\% ihrer Ressourcen auf interne Ermittlungen verwenden sollte (235). Dabei übertreibt er es natürlich nicht mit der Kritik an der üblichen Polizeipraxis und stellt klar: »Vielleicht wird einem das heute keiner mehr sagen, aber wenn man gegen einen Polizisten die Hand erhebt oder ihm ins Auge spukt, dann muss man damit rechnen, niedergeschlagen zu werden.« (2 I 8).

\section{Die Welt des Jack Maple}

Wie aus den bisherigen Ausführungen und Zitaten hervorgegangen sein mag, gibt es in der Welt des Jack Maple eine sehr klar gezogene Grenze, die trennscharf zwischen dem Crime Fighter und allen anderen verläuft. Diese Einteilung der Welt unterscheidet sich leicht, aber nicht unerheblich von der üblicherweise von US-amerikanischen Polizisten vorgenommenen Dichotomie zwischen Uns, der Polizei, und Ihnen, worunter 
nicht nur Verbrecher, sondern alle Zivilisten, Politiker und die Öffentlichkeit insgesamt fallen. Am deutlichsten wird diese strikte Grenze, wenn sie als Blue Wall of Silence die Ermittlungen bei Fällen von Polizeikorruption und -brutalität unmöglich macht (Chin, Wells 1998; Koepke 2000). Grundlage für diesen Verhaltenscode ist die in den USA besonders deutlich ausgeprägte Subkultur der Polizei, wie sie, zugegebenermaßen drastisch, zusammengefasst wird in der Aussage eines Police Officers:

If we're going to catch these guys, $\mathrm{f}^{* * *}$ the Constitution, $\mathrm{f}^{* * *}$ the Bill of Rights, $\mathrm{f} * * *$ them, $\mathrm{f} * * *$ you, $f^{* * *}$ everybody. The only ones I care about are my partners. (Baker $1985: 247 \mathrm{f}$.)

Die Trennlinie verläuft also zwischen denjenigen, die einen Cop bei Übertretungen decken, und denen, die etwas gegen diese Übertretungen haben. Dass solche Phänomene nicht der Vergangenheit angehören, zeigten die Ermittlungen der Christopher Commission in Los Angeles (I991) und der Mollen Commission in New York (1994), die Korruption, Übergriffe und eine hermetisch abgeschlossene Subkultur der Polizei zu Tage förderten.

Wie schon seine Ausführungen zur Bekämpfung derartiger Verhaltensweisen zeigen, hat Maple mit dieser Art von >Wir gegen den Rest $<$ nichts am Hut. Seine Trennlinie verläuft anders. Auf seiner Seite wähnt er etwa auch die Medien (169-172) und die Öffentlichkeit (239-240) - bzw. Teile dieser, so lange diese nämlich - und diese Tautologie stört ihn nicht - auch wirklich auf seiner Seite, der Seite des Crime Fighter, stehen und sich in den Dienst seiner Sache stellen (lassen). Nicht auf dieser Seite stehen hingegen auch all jene Polizisten, die nicht mit Leidenschaft bei der Sache, die eben keine Crime Fighter sind:

Es ist dasselbe in allen Police Departments: 40 Prozent der Truppe verstecken sich hinter ihren Schreibtischen. Weitere $4 \circ$ Prozent machen ihre Arbeit kompetent, aber ohne Leidenschaft und ohne viel zu bewirken. Zehn Prozent hassen den Job dermaßen, dass sie versuchen, alles Positive $\mathrm{zu}$ zerstören, das irgendjemand anderes zu erreichen versucht. Die letzten zehn Prozent betrachten den Job als Berufung; und das sind die zehn Prozent, die 90 Prozent der Arbeit machen. (7)

Die echten Crime Fighter sind »Idealisten und Innovateure « (IO), denen innerhalb der eigenen Organisation nur Steine in den Weg gelegt werden (6-Io) und die es mitunter gar mit ihnen übel meinenden Vorgesetzten zu tun bekommen (I $5-18$ ).

Doch ist der hauptsächliche Feind des Crime Fighter natürlich nicht der eigene Kollege und Vorgesetzte, sondern der Kriminelle bzw., wie Maple ihn nennt, der »böse Junge« (»bad guy«), »Gauner« (»crook«; Gegenstand der crookology, etwa: Wissenschaft vom Gauner; 35-63), »Bucklige (»hump«; 208) oder auch das »Raubtier« (»predator«; 95 u. ö.). Es gilt also, sich an dieses Tier anzuschleichen (»stalking the predatory criminal «; 35) und es zu jagen; gegen diesen Feind muss ein Krieg geführt werden. Damit sind Maples bevorzugte Analogien zur Polizeiarbeit genannt: die Tierwelt und der Krieg.

Zunächst zum Krieg: Laut Maple gäbe es viel zu lernen von Kriegsherren wie Rommel, Sun Tzu, Hannibal, Nelson, Patton oder Napoleon (I34-I40), vor allem wenn es um »Menschenführung, Einsatzplanung und strategisches Denken geht « (138). Zu der in den USA üblichen rhetorischen Analogie von Verbrechensbekämpfung und Krieg (waron crime, war on drugs) kommt bei Maple also noch hinzu, dass er ganz explizit Klassiker der Kriegsführung und Lehrbücher der Marines heranzieht, um seine Strategien zu begründen. Es ist klar, dass Polizeiarbeit, die wie Krieg betrachtet und betrieben wird, einem äußeren Gegner gegenübertritt, der zu vernichten und auf den keine Rücksicht zu nehmen ist. Dieser Gegner ist auch nicht mehr Teil derselben imaginierten Gemeinschaft oder Nation, sondern ein ihr äußerlicher Feind. Auch ist klar, dass so Methoden legitimiert werden, die aus der Praxis der 
Kriegsführung kommen und in den USA seit den I980er Jahren in Form der SWATTeams auch bei der Polizei zunehmend Realität werden. Diese Einheiten »betrachten Polizeiarbeit als Krieg « (Parenti I999: I I2) und greifen entsprechend auf »Ausrüstung, Ausbildung, Rhetorik und Taktiken der Kriegsführung « zurück (ebd.; zur Militarisierung der Polizei in den USA vgl. ebd. I I I-I 38; Kraska, Kappler I997; zur Kriegsrhetorik in New York: Brüchert, Steinert 1997).

Schließlich zur Tierwelt: Mit dieser Analogie wird der Verbrecher nicht nur aus der eigenen Gemeinschaft/Nation, sondern gar aus der eigenen Spezies ausgeschlossen. Eine »brauchbare Metapher für intelligente Polizeiarbeit ist die Jagd «(38), denn auch die Polizei ist hinter Raubtieren her. Nun hat sich in den USA die Bezeichnung "predator«, also Raubtier, auch für Verbrecher eingebürgert. Doch Maple geht über diese unbedachte sprachliche Gleichsetzung weit hinaus, wenn er von den »Wasserlöchern« (40) der Verbrecher spricht, davon, dass »bei einigen Verbrechen die Ähnlichkeiten zu dem, was man in Tierdokumentationen im Fernsehen sieht, nicht zu übersehen sind « (4I), oder ausführt, dass »Räuber, Vergewaltiger und Serienmörder [...], Einbrecher, Taschendiebe und selbst Farbschmierer [»scam artists «][...] die Logik des Raubtiers [benutzen] (38). Zu den Aufgaben des guten Polizisten gehöre es nun, die »Körpersprache des Wolfsrudels « (38) (d. h. eine Gruppe von Kriminellen an ihrem Gang) zu erkennen sowie den Augenblick, »in dem das Raubtier sich auf sein Ziel ausrichtet [...]. Wer jemals eine Katze bei der Jagd beobachtet hat, das ist der Augenblick, in dem die Augen sich ganz auf einen Punkt konzentrieren, der Kopf sich senkt, die Schultern angezogen werden und das Tier in ein langsames Kriechen verfällt« (52). Diese Gleichsetzung von Verbrechern mit Raubtieren leistet bei der Frage, wie Verbrecher sind und wie mit ihnen zu verfahren sei, manchen ideologischen Dienst. Der Verbrecher, so suggeriert sie, kann nicht anders, als das Gesetz zu übertreten, es ist Teil seiner Natur. ${ }^{6}$ Er ist somit als ganze Person, unabhängig von dem, was er außer Verbrechen noch so tut, der Feind und muss mit allen Mitteln zur Strecke gebracht werden. Der Verbrecher hat schließlich, wie das Tier, keine Gnade verdient, immerhin sind ihm menschliche Eigenschaften offenbar fremd.

Die Welt des Jack Maple ist also recht übersichtlich eingeteilt: Entweder Du gehörst zu uns, den Crime Fighters, bzw. unterstützt uns - dann bist Du auf der richtigen Seite -, oder Du störst uns bzw. gehörst gar zu denjenigen, die von uns gejagt werden - dann stehst Du auf der falschen Seite und wirst die Folgen zu tragen haben. Nun könnte man diese soziologischen Ausführungen als unwichtiges Begleitgeschwätz eines auf ein Zusatzeinkommen durch Buchverkauf aus seienden Polizeipraktikers abtun, dem es eigentlich nur um die in I. besprochenen Taktiken geht und der, um ein Buch zu füllen, einen Ghostwriter beauftragt hat, noch einige Seiten mit allgemeinen Betrachtungen zu füllen, die von niemandem wirklich ernst genommen werden. Dass Maples Modell der nach ihrer Nähe bzw. Distanz zu den Crime Fighters stratifizierten Gesellschaft so einflussreich sein könnte, wie seine Ausführungen zur Polizeitaktik, ist tatsächlich nicht anzunehmen. Eine andere Frage jedoch ist, warum gerade dieses Gesellschaftsbild in Kombination mit den angepriesenen Polizeistrategien auftaucht und offenbar gut dazu passt.

6 Ohne explizit eine naturdeterminierte Devianz zu behaupten, reiht sich Maple damit implizit in einen in der US-amerikanischen Kriminologie zu beobachtenden Trend ein, in der »Diskussionen um neuere biosoziale Untersuchungen erheblich zugenommen« haben (Kaiser 1998: 546). 
Wie kommt Maple auf seinen Maßstab zur Einteilung der Welt? Bei der Frage, warum ausgerechnet er Mitglied im kleinen Kreis der Crime Fighter wurde, sinniert er: »Vielleicht war es die Erwartung, dass [mein] Aufstieg durch die Ränge [...] sich auf lange Sicht einstellen würde, weil die Chefs immer ein paar Leute um sich herum brauchen würden, die den Laden tatsächlich schmeißen können« (ı)). Zum Crime Fighter wurde Maple demnach aus persönlichen (Karriere-)Motiven, sein Maßstab zur Einteilung der Welt leitet sich mithin aus dem Standpunkt seines eigenen Interesses ab. Dies ist weder verwerflich noch irgendwie ungewöhnlich, sondern vielmehr die dem bürgerlichen Individuum eigene Sicht der Dinge: »Alle Einrichtungen der kapitalistischen Welt und jeden >Mitmenschen $<$ betrachtet es als Bedingung seines eigenen Fortkommens, wobei ihm manches positiv, manches negativ vorkommt.«(Held I98I: is) Positiv kommen Maple demnach alle vor, die ihn bei der Verbrechensbekämpfung/seiner Karriere unterstützen, negativ alle, die dem entgegenstehen. Allerdings sind Maples private Karriereplanungen an sich natürlich nicht der Grund, warum er mit seiner Einteilung der Welt so weit gekommen ist. Um dies zu erklären, hilft ein Blick auf die sich verändernde Rolle der Polizei in der USA.

Maples Aufstieg begann zu einer Zeit, Anfang der i99oer Jahre, zu der eine grundlegende »Veränderung im Glauben an die Effektivität der Polizei« (Heymann 2000: 407) stattfand. Der Inhalt des neuen Glaubensbekenntnisses ist zusammengefasst im Titel eines Beitrages von William Bratton: Crime is Down in New York City: Blame the Police (1998a). Es geht also um den Anspruch der Polizei, für den Rückgang der erfassten Kriminalität in den i99oer Jahren verantwortlich zu sein (Kelling, Bratton I998; Bratton 1998; Kelling 2000). Dieser Anspruch widerspricht der Einschätzung von Kriminologie und Kriminalpolitik vorheriger Jahre, nach der die Entwicklung der Kriminalitätsziffern gesellschaftliche Gründe hat, auf die die Polizei kaum bis keinen Einfluss hat (außer, wie von Seiten des labeling approach betont, durch ihre Erfassungspraktiken). Zwischen den Vertretern der beiden Positionen ist in den I990er Jahren ein erbitterter Streit ausgebrochen. Die >gesellschaftlich ‘ argumentierende Seite führt dabei vor allem zweierlei Typen empirischer Belege an. Erstens sei in verschiedenen (wenn auch nicht allen) Städten der USA ein Kriminalitätsrückgang zu verzeichnen, und zwar unabhängig von der jeweils verfolgten Strategie (Joanes 2000; im Vergleich zu San Diego: Green I999; im Vergleich zu Boston: Berrien, Winship I999; O. A. I999; im Vergleich zu Kanada: Onimet 2002). Zweitens wird auf genuin gesellschaftliche Faktoren verwiesen, auf die die Polizei keinen Einfluss habe (Fagan, Zimring, Kim 1998). Dabei werden die demographische Entwicklung, die Entwicklung des Arbeitsmarkts, die hohe Einsperrungsrate und das Ende der Crack-Epidemie (Curtis 1998; Johnson, Golub, Dunlap, 2000) genannt. Wie Vertreter beider Seiten einräumen, ist dieser Streit weder entschieden, noch lässt er sich überhaupt empirisch entscheiden (Kelling 2000: 567; Greene I999: I78; Zimring, Fagan 2000: 450). Wichtig jedoch ist der Umstand, dass der Polizei in den öffentlichen Debatten überhaupt wieder zugetraut wird, die Kriminalität zu bekämpfen, d.h. als Crime Fighter aufzutreten. Franklin Zimring und Jefferey Fagan sprechen in diesem Zusammenhang von einem »beinahe euphorischen Wettbewerb der Ansprüche, den Rückgang der Kriminalität auf bestimmte Gegenmaßnahmen zurückzuführen«(2000: 455). Speziell für New York hat Sylke Nissen in einem Vergleich der dortigen Stadtpolitik mit der in London und Berlin gezeigt, wie deren Erfolg auch und vor allem mit Giulianis »Agenda-Setting-Kompetenzen« zu tun hat, die ihn in die Lage versetzte, »seine Sicht der New Yorker Realität im Bewusstsein der Öffentlichkeit zu implementieren « (2002: 2 I ). Inhalt dieser Sicht war: Ich und meine Polizei machen New York sicherer. 
In eben diesem Kontext wird Personal benötigt, das, wie Maple, an eben diese Aufgabe der Polizei auch tatsächlich glaubt. ${ }^{7}$

Wie einige der Chefideologen der neuen Polizeistrategien betonen, ist die Entscheidung der Glaubensfrage, ob man der Exekutive die Eindämmung des Verbrechens zutraut oder nicht, nicht zuletzt abhängig von der eigenen Positionierung innerhalb der US-amerikanischen Culture Wars (Kelling 2001; Wilson 1996). Mit diesem Schlagwort werden üblicherweise die Auseinandersetzung zwischen (im US-amerikanischen Sinn) Konservativen und Liberalen bezeichnet, die sich an Fragen wie Abtreibung (Moww, Sobel 200I), Lehrplänen öffentlicher Schulen (Merrett 1999), Drogenpolitik u.v.a.m. entzünden. Bei der Verbrechensbekämpfung gibt es, wie Theodore Caplow und Jonathan Simon zur Erklärung der Beliebtheit dieses Themas in der US-amerikanischen Politik ausführen, grundsätzlich weit weniger Streit, sie ist »eine der wenigen Quellen von Einigkeit in einer zerstrittenen Öffentlichkeit« (Caplow, Simon 1999: 79). Umstritten ist hier vielmehr die Frage der richtigen Methoden. In seinem einflussreichen Buch Culture Wars führt James Hunter (I99I) die verschiedenen Konflikte zwischen »Orthodoxen« und »Progressiven« auf die Frage zurück, ob diese bei Fragen der Moral eine transzendentale Autorität als höchste Instanz annehmen oder sie entsprechend der »vorherrschenden Annahmen des Alltagslebens « (ebd.: 45) entscheiden. Anders James Q. Wilson, der den entscheidenden Unterschied zwischen den Kriegsparteien in der Betonung von individueller Freiheit vs. Gemeinschaft (»community«) sieht (Wilson 1996). Gemeinsam ist diesen Erklärungsversuchen, dass sie die Differenz der beiden Kriegsparteien in kulturellen, mithin ideellen Positionen suchen und damit von jeglicher materieller Grundlagen der Auseinandersetzungen abstrahieren.

Einen anderen Weg schlägt etwa der Geograph Don Mitchell (2000) in seiner Cultural Geography ein: Um sich nicht in der kulturalistischen Tautologie zu verstricken, in der kulturelle Auseinandersetzungen mit kulturellen Unterschieden erklärt werden, gälte es erstens zu verstehen, »was Kultur ist und was nicht «(2000: I2), und zweitens zu erklären, »wie das, was Kultur genannt wird, selbst durch Konflikt und Kooperation über die Materialien [...], die unser Leben ausmachen, sozial konstruiert wird « ( $e b d$.). Während er zum ersten Punkt ausführt, dass es sich bei »Kultur« um Ideologie handelt, die, obschon nicht auf die Zirkulation von Kapital und Waren reduzierbar, von dieser auch nicht zu trennen sei (Mitchell 2000: 80; vgl. dazu Harvey 1989), gilt es bei dem zweiten Punkt bei den jeweiligen scheinbar kulturellen Konflikten nach deren materieller Grundlage zu suchen. Für die Frage der Effektivität der Polizeiarbeit sei diese Suche abschließend angedeutet.

Wie vor allem die Ideologen der neuen Polizeistrategien betonen, gehört zu den zentralen Annahmen einer erfolgreichen Bekämpfung des Verbrechens, dass erstens die gesamte Gesellschaft mobilisiert wird, um zweitens die öffentliche Ordnung aufrecht zu erhalten. Und obschon sie nicht allein für Sicherheit und Ordnung verantwortlich sei, komme dabei der Polizei eine zentrale Rolle zu. Diese beiden Aspekte - >gesamtgesellschaftliche Aufgabe < und `öffentliche Ordnung< - sind allen neueren Ansätzen der Polizeiarbeit (einschließlich der Wiederentdeckung der Prävention, vgl. Frehsee 1998; Sack 1995) gemein und bedeuten eine Ausweitung der Zuständigkeit der Polizei, ganz gleich ob unter dem Label zero tolerance bzw. broken windows oder, als scheinbarem Gegenentwurf, ${ }^{8}$ community policing (Skogan, Hart-

7 Für Maple ist in aller Selbstverständlichkeit ausschließlich die Polizei für die Entwicklung der gemessenen Kriminalitätsbelastung verantwortlich: » 1980 , als die Zahl der Überfälle ihren höchsten Stand ereichte, waren es täglich fast 300. Die Cops konnten das zur Mitte des Jahrzehntes bremsen, aber ein laxes enforcement ließ die Ziffer wieder auf rund 275/Tag im Jahr I 990 anwachsen.« (53)

8 Zwischen zero tolerance und community policing existieren zwar Unterschiede, weshalb eine vergleichende 
nett I997, insb.: I 62). Wenn nun die öffentliche Ordnung wiederentdeckt wird, »dann

öffnet sich die Staatsmacht « - in der pointierten Formulierung Detlev Frehsees »damit die Tore zur Reglementierung von Fragen des Benehmens, der Höflichkeit und Rücksichtnahme, des guten Geschmacks, der Ästhetik, der Sauberkeit, des Lebensstils « (2000: 66). Diese Vorverlagerung des staatlichen Eingriffs in die Privatsphäre, die immer mehr ohne konkrete Verdachtsmomente auskommt, und ihre Verrechtlichung hat Fritz Sack als »neue Prävention« bezeichnet. Diese »transformiert ein reaktives Strafrecht in ein proaktives System strafrechtlicher Sozialkontrolle. Nicht mehr die Reaktion auf erfolgte Regelverletzungen, sondern deren Antizipation, Vorauserkennung, Vorankündigung [...] markieren die Perspektiven einer so umgestalteten staatlich-strafrechtlichen Sozialkontrolle«(Sack i 995: 45 I f.).

Ziel diese >net-widening ${ }^{9}$ ist die möglichst weitgehende Kontrolle aller Lebensbereiche. Im Zentrum steht dabei ein Staat, der alles im Griff hat - oder dies zumindest vorgibt, auch wenn er sich verabschiedet hat vom »Mythos, [...] >law and order innerhalb seines Territoriums garantieren zu können« (Garland 2001: I09). Diese Wiederkehr des starken, strafenden Staates (Hansen 1999) folgt auf eine Phase, in der soziale Kontrolle stärker durch die Inklusion beinahe aller Teile der Bevölkerung in den expandierenden Wohlfahrtsstaat bewerkstelligt werden sollte (Beckett, Western 200I; Garland 200 I; Wacquant I997). Diese eher inklusive Strategie hatte ihren Grund in dem tendenziellen Arbeitskräftemangel und, speziell in den USA, der Furcht vor sozialen Unruhen (Piven, Cloward I993). Nachdem beides seit den I980er Jahren nicht mehr zutrifft, steht nunmehr ein War Against the Poor (Gans 1995) auf der Tagesordnung, in dem die Hilfe bei Armut durch die Kriminalisierung von Armen ersetzt wird. Dieser Krieg hat auch eine Geographie: Er wird dort geführt, wo diese Armen (vorwiegend Afroamerikaner und Hispanics) zu finden sind: in den inner cities der großen Städte, den »Ghettos der Ausgeschlossenen«(Marcuse 1998: 192). Die Kriminalisierung dieser Gruppen und Orte wird legitimiert mit einer Ideologie, die David Garland als die »Kriminologie des Anderen « (200I: I 37) bezeichnet hat. Dieser »durch und durch praktische und atheoretische« (ebd.: I 27) Diskurs stellt für ihn das »kriminologische Echo von Culture Wars und neokonservativer Politik (ebd.: I 84) dar. In ihr wird »der bedrohliche Outcast, der angsteinflößende Fremde, der Ausgeschlossene und der Verbitterte ( $e b d .:$ I 37$)$ zu einer »Person, die sich jenseits aller Grenzen, jenseits aller Reform, außerhalb der zivilisierten Gemeinschaft befindet « (ebd.: I 85 ). Es sind dies diejenigen, um die sich zu kümmern der Staat nicht mehr für notwendig erachtet und die nur mehr (möglichst billig) kontrolliert werden sollen; diejenigen, die deshalb in Politik und Medien dämonisiert und diskursiv aus der Gemeinschaft der >law abiding citizens < ausgeschlossen werden; diejenigen, die in den

Gegenüberstellung der beiden Ansätze natürlich legitim ist (etwa: Legge 1997, insb. I I7), doch sind diese, wie Joachim Kersten (2002) betont, »kein reales Abbild polizeilicher Tätigkeit«(S. 98). M. E. sind vor allem zahlreiche Gemeinsamkeiten festzuhalten; neben den beiden genannten - Einbeziehung weiterer Akteure und Betonung der öffentlichen Ordnung - sind die Dezentralisierung der Polizeiarbeit und die Betonung der Öffentlichkeitsarbeit zu nennen. Zudem ist aus der Sicht der Vertreter der >harten Linie ihr Ansatz ebenfalls community policing (Bratton 1998; Kelling 200I: I4I, wo er behauptet, community policing in einem Aufsatz von 1978 erfunden zu haben).

9 Mit dem Bild des snet-widening bezeichnet Cohen (1985) die immer weiter um sich greifende soziale Kontrolle durch den Staat. Die Frage, ob in den vergangenen Jahrzehnten in den USA ein solcher Prozess stattgefunden hat, oder ob nicht vielmehr Entkriminalisierungstendenzen in die entgegengesetzte Richtung weisen, ist ebenfalls Gegenstand von Auseinandersetzung. Neokonservative wie Wilson und Kelling (1982) etwa beklagen die Entkriminalisierung verschiedener Verhaltensweisen im öffentlichen Raum (vgl. auch Siegel 1995). Doch auch etwa David Garland (200I: I 17-I 19) will das Bild des >net-widening< relativiert wissen, da die administrative Reaktion auf das - von ihm konstatierte - Aufkommen von Kriminalität als Massenphänomen vor allem im Herunterdefinieren von Devianz bestanden habe. Diese Einschätzung wird jedoch von Katherine Beckett (200I) überzeugend kritisiert. 

gegner und Raubtiere bekämpft und gejagt werden.

So erfüllt seine Lobpreisung des Crime Fighting auch und vor allem eine ideologische Funktion, kann man doch mit Sidney Harring feststellen, dass die Polizei »ohne ihre legitimierende Crime Fighter-Rolle schlicht gar keine Funktion erfüllen [könnte] (1983: 246). Anders formuliert: Ganz ohne Legitimierung kommt die Kontrolle der Überflüssigen durch die Polizei heute ebenso wenig wie in der von Harring untersuchten Zeit der Industrialisierung aus, weshalb sie als Verbrechensbekämpfung dargestellt wird. So war Maple für jene, die in den Culture Wars betonen, dass die Polizei die Kriminalität durch hartes Durchgreifen bekämpfen kann, und die doch eigentlich der »Kontrolle von armen Leuten in armen Gegenden «(Fagan und Davies 2000: 457; s. o.) qua Wiederherstellung der öffentlichen Ordnung das Wort reden, der perfekte Schlachtherr: jemand, dessen Strategien einem Weltbild entsprechen, in dem die Inner City Poor nicht mehr zur Nation bzw. zur menschlichen Spezies gehören und durch die Crime Fighters überwacht werden.

\section{Literatur}

Baker, M. (1985): Cops. Their Lives in Their Own Words. New York.

Beckett, K. (200I): Crime and Control in the Culture of Late Modernity. In: Law \& Society Review 35. Jg. S. 899-929.

Dies. (1997): Making Crime Pay. New York und Oxford.

Dies. und Western, B. (200I): Governing social marginality. Welfare, incarceration, and the transformation of state policy. In: Garland, D. (Hrsg.): Mass Imprisonment. London et al, S. $35-50$.

Belina, B. (2000): Kriminelle Räume (= Urbs et Regio 7I). Kassel.

Ders. (2000a): »Kriminalität« und »Raum«. In: KrimJ 32. Jg. S. I29-I 47.

Ders. und Helms, G. (2003): Zero Tolerance for the industrial past and other threats: Policing and urban entrepreneurialism in Britain and Germany. In: Urban Studies 40. Jg., S. I8451867.

Berrien, J. und Winship, C. (1999): Lessons Learned from Boston's Police-Community Collaboration. In: Federal Probation 63. Jg. H. 2 S. $25-32$.

Bouza, A. (200I): Police Unbound: Corruption, Abuse, and Heroism by the Boys in Blue. New York.

Ders. (1990): The Police Mystique. An Insider's Look at Cops, Crime, and the Criminal Justice System. New York und London.

Bratton, W. (with P. Knobler)(1998): Turnaround. New York.

Ders. (1998a): Crime is Down in New York City: Blame the Police. In: Dennis, N. (Hrsg.): Zero Tolerance. 2. Aufl. London, S. 29-43.

Brüchert, O. und Steinert, H. (1997): Popularisierung der sozialen Ausschließung: Zwischen Kriegsmetaphern und Strafwünschen. In: Widersprüche 17. Jg. H. 4, S. I63-I77.

Caplow, T. und Simon, J. (1999): Understanding Prison Policy and Population Trends. In: Tonry, M. und Petersilia, J. (Hrsg.): Prisons (= Crime and Justice 26). Chicago, S. 63-I 2 I.

Chin, G. und Wells, S. (1998): The »Blue Wall of Silence« as Evidence of Bias and Motive to Lie. In: University of Pittsburgh Law Review 59. Jg. S. 233-299.

Cohen, S. (1985): Visions of Social Control. Cambrigde.

Cole, D. (1999): No Equal Justice. New York.

Curtis, R. (1998): The improbable transformation of inner-city neighborhoods: crime, violence, drugs, and the youth in the I990s. In: Journal of Criminal Law and Criminology 88. Jg. S. I $233-1276$.

Fagan, J., Davies, G. (2000): Street Stops and Broken Windows: Terry, Race, and Disorder in New York. In: Fordham Urban Law Journal 28. Jg. S. 457- 504.

Fagan, J., Zimring, F., Kim J. (1998): Declining Homicide in New York City: A Tale of Two Trends. In: Journal of Criminal Law and Criminology 88. Jg. S. I 277-1 323.

Fischer, G., Roth, J. (1998): Leben voller Fallrückzieher. Leipzig.

Frehsee, D. (2000): Fragen an den Deutschen Präventionstag. In: DVJJ-Journal I I. Jg. Nr. I67, S. $65-72$.

Ders. (1998): Politische Funktionen Kommunaler Kriminalprävention. In: Albrecht, H.-J. u. a. 
(Hrsg.): Internationale Perspektiven in Kriminologie und Strafrecht. Festschrift für Günther Kaiser. I. Halbband. Berlin, S. $739-763$.

Gans, H. (1995): The War Against the Poor. New York.

Garland, D. (200I): The Culture of Control. Oxford.

Gehring, I. (I 999): »Innere Sicherheit - USA «. Rechtsvergleich der Entwicklung, Organisation, Aufgaben der Polizei und deren Kompetenzen (= Würzburger Rechtswissenschaftliche Schriften I7). Würzburg.

Greene, J. (1999): Zero Tolerance: A case study of police policies and practices in New York City. In: Crime \& Delinquency 45. Jg. S. I7 I-I 87.

Hansen, R. (1999): Eine Wiederkehr des »Leviathan «? Starker Staat und neue Sicherheitsgesellschaft. In: KJ 32. Jg. S. 23 I-253.

Hard, G. (1987): »Bewußtseinsräume«. Interpretationen zu Versuchen, regionales Bewußtsein zu untersuchen. In: Geographische Zeitschrift 75. Jg. S. 21 7-I 48.

Harring, S. (1983): Policing a Class Society. New Brunswick.

Ders. und Ray, G. (1999): Policing a Class Society: New York in the 1990s. In: Social Justice 26. Jg. S. 63-8I.

Harvey, D. (1989): The Condition of Postmodernity. Oxford.

Held, K. (Hrsg.)(198I): Die Psychologie des bürgerlichen Individuums. München.

Hess, H. (1999): Fixing Broken Windows and Bringing Down Crime. In: KJ 32. Jg. S. 32-57.

Heymann, P. (2000): The New Policing. In: Fordham Urban Law Journal 28. Jg. S. 407-456.

Hickman, M. und Reaves, B. (200I): Local Police Departments I 999 (Bureau of Justice Statistics, Law Enforcement Management and Administrative Statistics).

Hunter, J. (1991): Culture Wars: The Struggle to Define America. New York.

Joanes, A. (2000): Does the New York City Police Department Deserve Credit for the Decline in New York City's Homicide Rates? In: Columbia Journal of Law and Social Problems 33. Jg. S. 264-3II.

Johnson, B., Golub, A. und Dunlap, E. (2000): The Rise and Decline of Hard Drugs, Drug Markets, and Violence in Inner-City New York. In: Blumstein, A., Wallman, J. (Hrsg.): The Crime Drop in America. New York. S. 164-206.

Jones, R. (2002): Crimson Street. In: Baltimore Times, 5. 04. 2002.

Kaiser, G. (1998): Was heißt »Amerikanische Kriminologie«? In: Scbwind, H. u. a. (Hrsg.): Festschrift für Hans-Joachim Schneider. Berlin und New York, S. 539-563.

Kelling, G. (200I): >Broken Windows< and the culture wars. In: Matthews, R. und Pitts, J. (Hrsg.): Crime, Disorder and Community Safety. London und New York, S. I 20-I 44.

Ders. (2000): Why did people stop committing crimes? An essay about criminology and ideology. In: Fordham Urban Law Journal 28. Jg. S. $567-586$.

Ders. und Bratton, W. (1998): Declining Crime Rates: Insiders' Views of the New York City Story. In: Journal of Criminal Law and Criminology 88. Jg. S. I 2 I7-I 23 I.

Kersten, J. (2002): Zero Tolerance oder Community Policing. In: Neue Kriminalpolitik I4. Jg. S. $94-99$.

Klüter, H. (1986): Raum als Element sozialer Kommunikation (= Giesener Geographische Schriften 60). Giesen.

Koepke, J. (2000): The Failure to Breach the Blue Wall of Silence: The Circling of the Wagons to Protect Police Perjury. In: Washburn Law Journal 39. Jg. S. 2 I I-24I.

Kraska, P. und Kappler, V. (1997): Militarizing American police: the rise and normalization of paramilitary units. In: Social Problems 44. Jg. S. I-I 8.

Legge, I. (1997): New York - weder Modell noch Fortschritt? In: Dreher, G. und Feltes, T. (Hrsg): Das Modell New York: Kriminalpolitik durch »zero tolerance«? Holzkirchen, S. $102-199$.

Lock, E., Timberlake, J. und Rasinski, K. (2002): Battle Fatigue: Is Public Support Waning for »War«-Centered Drug Control Strategies? In: Crime \& Delinquency 48. Jg. S. 380-398.

Marcuse, P. (1998): Ethnische Enklaven und rassische Ghettos in der postfordistischen Stadt. In: Heitmeyer, W., Dollase, R. und Backes, O. (Hrsg.): Die Krise der Städte. Frankfurt a. M., S. $176-193$.

Merrett, C. (1999): Culture Wars and National Education Standards: Scale and the Struggle over Social Reproduction. In: Professional Geographer 51. Jg. S. 598-609.

Mitchell, D. (2000): Cultural Geography. Oxford.

Mouw, T. und Sobel, M. (200I): Culture Wars and Opinion Polarization: the Case of Abortion. In: American Journal of Sociology 106. Jg. S. 91 3-943.

Nissen, S. (2002): Die regierbare Stadt. Opladen.

O. A. (1999): First safety, then civility. New York's troubled police force can learn from its counterpart in Boston. In: The Economist, I. 5. 1999. 
O'Malley, M. und Norris, E. (2000): The Mayor's Plan to Dramatically Reduce Crime in Baltimore. Baltimore.

Ouimet, M. (2002): Explaining the American and Canadian crime »drop« in the I990's. In: Canadian Journal of Criminology 44. Jg. S. 33-50.

Parenti, C. (1999): Lockdown America. London und New York

Piven, F. und Cloward, R. (I993): Regulating the Poor. Updated edition. New York [1971].

Rath, M. (2000): Command Decisions. In: Baltimore City Paper, I9. 04. 2000.

Redmond, H. (200I): The War on Drugs. Myth and Reality. In: International Socialist Review H. Is December 2000 - January 200 I, S. 35-42.

Sack, F. (I995): Prävention - ein alter Gedanke in neuem Gewand. In: Gössner, R. (Hrsg.): Mythos Sicherheit. Baden-Baden, S. 429-456

Siegel, F. (1995): Reclaiming our Public Spaces. In: Kasinitz, P. (Hrsg.): Metropolis. New York, S. $369-383$.

Skogan, W. und Hartnett, S. (1997): Community Policing, Chicago Style. New York und Oxford.

Smith, N. (200I): Global Social Cleansing: Postliberal Revanchism And the Export of Zero Tolerance. In: Social Justice 28. Jg. S. 68-74.

Wacquant, L. (2000): Elend hinter Gittern. Koblenz.

Ders. (1997): Vom wohltätigen Staat zum strafenden Staat: Über den politischen Umgang mit dem Elend in Amerika. In: Leviathan 25. Jg. S. 50-66.

Wilber, D. (200I): Reinventing police work. In: Baltimore Sun, I 2. 08. $200 \mathrm{I}$.

Wilson, J. (1996): Foreword. In: Kelling, G., Coles C.: Fixing Broken Windows. New York u. a., S. xiii-xvi.

Ders. und Kelling, G. (1982): Broken Windows. In: Atlantic Monthly H. 3 S. 29-38.

Zimring, F. und Fagan, J. (2000): The Search for Causes in an Era of Crime Declines: Some Lessons From the Study of New York City Homicide. In: Crime \& Delinquency 46. Jg. S. $446-456$.

Zurawik, D. (2002): Commissioner brings credibility to >Wire role. In: Baltimore Sun, 23. 8. 2002 .

\section{Henry Friedlander Das Novemberprogrom und die Justiz nach 1945} Eine Fallstudie

This essay deals with a criminal trial, part of my current research into the postwar trials of the Kristallnacht perpetrators. These were not Allied trials, but proceedings in the newly reconstituted German courts. ${ }^{\text {I }}$ There were hundreds of Kristallnacht trials covering every region of Germany and Austria; they commenced in late I945 and petered out in the early i 950 s. For this essay, I have selected a case from the holdings of the Staatsarchiv in Oldenburg, where it is listed as Case No. I I93. ${ }^{2}$

Case I 93 concerned an unusual Kristallnacht story. Actually, not a Kristallnacht story at all, although it is listed as such by the archive. The November pogrom took place on the 9 th and the roth; Case I 93 occurred on the evening of the I 5 th. More important, the November pogrom was directed against Jews; the victims of Case I 193 were not Jewish. It is the only such case I have found, and that makes it rather unusual.

* This is a revised version of my presidential address (Eine Berliner Pflanze-An Unusual Kristallnacht Story), delivered at the annual meeting of the German Studies Association, San Diego, 4 October 2002. It was published in German Studies Review XXVI/I (February 2003), pp. I-I4.

I See Henry Friedlander, "The Judiciary and Nazi Crimes in Postwar Germany, «in: Simon Wiesenthal Center Annual I (1984): 27-44.

2 Staatsarchiv Oldenburg, Bestand I40-5, Nr. I I 93: Verfahren Hartmann, 5 Js I I07/47, 9 Ks 2/50, 2 vols. 